\title{
Transformation of Industrial Enterprises in the Digital Economy
}

\author{
Sergey Novikov ${ }^{1}$ \\ ${ }^{1}$ Moscow Aviation Institute (National Research University), Moscow, Russian Federation \\ Correspondence: Sergey Novikov, Moscow Aviation Institute (National Research University), Moscow, 4, \\ Volokolamskoe Highway, 125993, Russian Federation. Tel: 7-963-753-7110. E-mail: danonik92@mail.ru, \\ ncsrm@mail.ru
}

Received: April 6, 2020

Accepted: May 16, 2020

Online Published: September 3, 2020

doi:10.5430/rwe.v11n5p90

URL: https://doi.org/10.5430/rwe.v11n5p90

\begin{abstract}
The article is devoted to the analysis of transformation processes of high-tech industrial enterprises operating in the digital economy. It is noted that in the modern economy of Russia the technologies of the third and fourth technological paradigm (TP) prevail, therefore the technologies of the fifth and sixth ones are "high" for it. The structure of the transformation processes of a high-tech enterprise in the framework of the development of digital technologies with the allocation of technological levels of automation of the production process is considered. To optimize the processes of technological transformation, the author proposes to use a modified structure. A characteristic feature of the proposed structure is the presence in it of the so-called "intelligent agents", which are specialized software modules that are controlled by a special data exchange protocol. Modules also have the ability to implement synchronization mechanisms with business objects and provide the technical ability to integrate three or more different information systems.
\end{abstract}

Keywords: digital economy, innovative industrialization, systemic paradigm, competitiveness, technological paradigm (TP)

\section{Introduction}

Modern industry is characterized by a desire for globalization. The business models of most enterprises are based on interaction with different regions: China, Europe, or North America. In the international market, the attractiveness of a company is characterized only by competitiveness. To reduce the cost of production or increase sales and market share, companies are investing in digitalization of enterprises. The number of digital transformation projects is actively growing in the most industrialized regions of the world and in countries technologically inferior to the leaders. At the same time, the industry does not have a clear understanding about the tasks, that specific technologies solve and how to maximize the economic effect of implementation. Each enterprise understands, what is it, "digital transformation", in its own way. So-called smart operations also have a big impact on enterprises. With their help, operational transparency is created, which allows combining departments and disparate assets into a single mechanism. At the same time, in the lagging companies, business units often are independent of each other and ignore priorities that can increase the productivity of the enterprise as a whole (Savich, 2018).

It is important to understand that digital transformation is not just the introduction of new solutions and technologies. These projects require a certain technological and organizational base. It will be impossible to create innovative production without appropriate training. The general logic is simple: we need to start trying, testing new tools, launch pilot projects on a separate production line, and then move on to the level of the plant, complex of enterprises, supply chain, etc. So, the process is gradually moving towards global digitalization of the corporation. The success of the transformation depends to a large extent on maturity and readiness for modernization. People have to work with new tools, so, the level of competence of the company's employees should correspond to the technologies being introduced. Equally important criterion is equipping the enterprise with modern means of automatic process control systems (APCS). They generate the bulk of the data that will become the foundation for digital production.

Digitalization brings changes not only to the business, but also to information technologies (IT) departments, which have to significantly revise their work strategy and change the paradigm of relations with internal customers. In the digital paradigm, IT is business, and business is IT. Based on the above phenomenological definition of digital business, IT is the root element for each of the listed features of a digital enterprise. It is almost impossible to determine the universal requirements for the knowledge and skills of IT specialists in a digital enterprise, but IT must 
be a new way of thinking and action for the successful implementation of digital initiatives. It is necessary to refuse the strictly regulated process and project-oriented activities and understand the most important thing is the continuous creation of a new business value and improving the user experience, we need to fully accept continuous changes, be bold and ready to make decisions, defend our point of view from the position of consumers of the enterprise's product, introduce innovations, and increase internal IT efficiency (Orekhova, 2018).

Hyper-converged infrastructure is one of the assistants of digital transformation and one of the most important indicators for doing business in the modern world. The desire of the business to reduce this indicator has led to the use of hyperconverged solutions, due to which it becomes possible "out of the box" to get the optimal, pre-configured infrastructure, easy to operate and scale. Hyperconverged infrastructure, on the one hand, is an analogue of the infrastructures used by big web companies, and on the other hand, the minimum size of the building block allows it to be implemented even by small enterprises, doing this in stages, without serious capital costs during the initial implementation. An absolute advantage of hyperconverged architecture is the ability to quickly manage the necessary IT resources, given the changing business requirements and external market factors. But the big business gets the most significant result from this thanks to the scale. Hyperconverged architecture and solutions based on it contribute to increasing the competitiveness of the enterprise; it also provides the opportunity to adapt the IT infrastructure to the current needs of the business and almost linearly increase productivity in accordance with the needs of the business. The second factor is controllability, i.e. at any time, it is possible to turn on or off the virtual machine and provide it with more or less resources.

Digital transformation leaders, in turn, are focused on changing business processes and adapting organizational structure. A characteristic feature of successful enterprises is an orientation towards the identification and restructuring of inefficient processes. In addition, the efforts of leading companies are focused on a specific task: improving the efficiency of production operations and enterprises and creating added value for customers through competitive advantages. About $80 \%$ of successful projects are related to timely investments in the continuous improvements. Leaders create a team within the organization that is busy constantly improving key indicators and making decisions on the relevance of introducing new tools. The level of understanding of the enterprise's business model, its main competitive advantages and potential to reduce costs and increase market share are the basis for the implementation of digital transformation.

\section{Methodology and Theoretical Basis}

Technological updating ("modernization" and "new industrialization") of the existing industrial complex of Russia is considered as one of the main conditions for achieving national competitiveness and, as a result, ensuring national security. Since 2000, the highest state level has repeatedly posed the task of technological renewal of the economy. At first, it was formulated as "modernization", then as "new industrialization" ("neo-industrialization", "reindustrialization"), in 2014 it was successfully transformed into "import substitution". Despite the difference in formulations, the solution to all these problems is possible only through the transition of the Russian industry to the production of higher technology products. "Modernization" involves the transition to higher technologies. "New industrialization" is the formation of new, that is, more technologically advanced, industries. "Import substitution" is the transition to the manufacture of products that are purchased in other countries as products of higher technologies (Ilyin, Levina \& Dubhorn, 2019).

TP is a certain structured holistic and sustainable thing, within which a closed cycle is carried out, starting with the extraction and obtaining of primary resources and ending with the release of a set of end products that are suitable for the type of public consumption. The core of TP forms a complex of basic aggregates of technologically related industries. Technological innovations that determine the formation of the core of TP are key factors. In turn, industries that intensively use a key factor and play a leading role in the dissemination of a new TP are the main industries.

The technologies of the third and fourth TP prevail in the modern economy of Russia, therefore the technologies of the fifth and sixth TP are "high" for it. In this regard, the solution of the problems of "modernization", "new industrialization", or "import substitution" is possible only by replacing the technologies and production of the third and fourth TP with the technologies and production of the fifth and sixth TP. This provides for the transformation of the composition, structure, and communication system of the industrial complex, within which the products of the third and fourth TP are made, into the composition, structure, and communication system of the industrial complex, within which the fifth and sixth TP will be produced. Now we are going to consider in more detail the core of the sixth TP and highlight its main components:

- nanoelectronics and nanochemistry;

- nanophotonics; 
- nanomaterials and nanostructured coatings;

- nanosystem technology;

- nanobiotechnology;

- IT;

- convergence of nano-, bio-, info- and cognitive technologies.

Now we are going to consider the main characteristics of industrial transformation (Gileva, 2019):

1. It is an integral part of the digital transformation and includes, among other things, the Industrial Internet of Things (IIoT). Industrial transformation is aimed at the production part of discrete, continuous, or mass production. Thus, the operational activities of plants, factories, and mines are in a direct area of influence of industrial transformation.

2. Industrial transformation provides full support for the concept of consistent production improvements.

3. One of the main tasks of industrial transformation is the coordination, management, and replication of decisions and adopted practices at remote production sites. Manufacturing is a geographically and organizationally distributed structure, often with sites that are hundreds or even thousands of kilometers distant from each other. Inhomogeneities and differences in the production culture of sites make standardization of processes difficult. In this case, one of the main tasks of industrial transformation is not only to find a suitable solution for a business problem, but also to ensure the possibility of its adaptation and replication taking into account the specifics of remote production sites.

4. Industrial transformation includes assets, processes, and enterprise products:

- asset-based initiatives include various areas for improving productivity and utilization of core production equipment;

- product initiatives include areas for improving the quality of the products manufactured by the enterprise and areas for reducing the volume of rejects or non-conforming products, and shortening the production cycle;

- initiatives aimed at operational activities include areas for improving the interfunctional interaction of various production departments, conversions, and divisions to increase the efficiency of production or providing services to customers.

Just having a digital transformation concept or roadmap is not a competitive advantage. We need funds that will provide the opportunity to achieve our goals and solve the problems of digital and industrial transformation. Also, the choice of a solution is not only in finding the right technology for implementation.

One of the most important characteristics of a modern digital enterprise is its integration, i.e. the presence of a corporate ecosystem that combines not only automation systems within the enterprise, but also two other equally important components: processes and staff. The mutual integration of processes, staff, and information systems allows to increase the return of each of the components, and, in general, improve the work of production redistribution due to the following (Dmitriev \& Novikov, 2019):

- digital work management;

- standardization of production processes based on corporate practices taking into account the specifics of individual production sites / divisions.

- collaboration of staff and automation systems from different departments.

- modular development, single user interface, and remote deployment of applications.

An integral element of the organization of such mutual integration is the business process management system (BPMS) of production, which provides the ability to digitally represent business processes adopted at the enterprise or best industry practices, horizontal (between units of the same level) and vertical (between different levels) integration staff (Koch \& Windsperger, 2017).

Information systems are a medium of exchange and perceive innovations, but for the majority of industrial enterprises, information systems are modernized through imitation, based on the use of already created samples of information systems, the effectiveness of which has already been confirmed, and they satisfy the existing system of requirements and provide information exchange within the enterprise and with counterparties. Especially important in modern conditions is that the imitation of information systems ensures the compatibility of enterprises in the 
globalization. As an example, there is the enterprise information management (EIM) system, which is built on the combination of enterprise resource planning (ERP), product lifecycle management (PLM), manufacturing execution systems (MES), and manufacturing data collection (MDC) and allows us to create a centralized digital information hub used at all stages of the life cycle of a production project. There is the nature of the transformation in relation to the selected subsystems of an industrial enterprise in the Figure 1.

The use of MES has been effective for the entire time since its introduction and active implementation in production. With the advent of new digital technologies, such as Big Data, IIoT, BPMS, and MES have gained additional opportunities to monitor the status of production and track inventory almost at the pace of production (Ananyin, Zimin, Lugachev, Gimranov \& Skripkin, 2018).

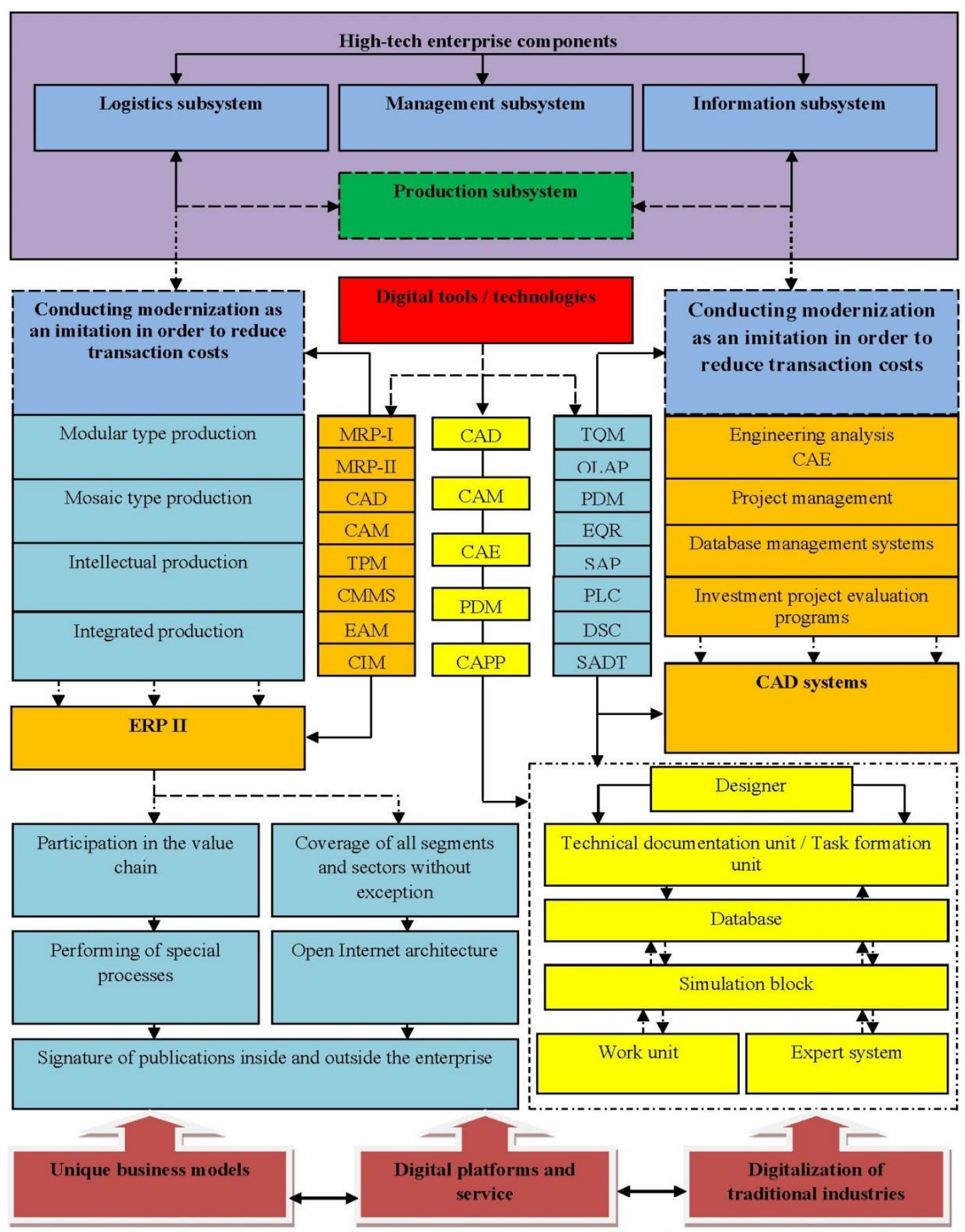

Figure 1. Transformation of a high-tech enterprise as a part of the development of digital technology 
Legend:

1. Material requirements planning (MRP-I);

2. Manufacturing resource planning (MRP-II);

3. Computer-aided design/drafting (CAD);

4. Computer-aided manufacturing (CAM);

5. Total productive maintenance (TPM);

6. Computerized maintenance management system (CMMS);

7. Enterprise asset management (EAM);

8. Common information model (CIM);

9. Computer-aided engineering (CAE);

10. Product data management (PDM);

11. Computer-aided process planning (CAPP);

12. Total quality management (TQM);

13. Online analytical processing (OLAP);

14. Product data management (PDM);

15. European qualification basis for lifelong learning (EQR);

16. Systems, applications and products in data processing (SAP);

17. Programmable logic controller (PLC);

18. Dynamic stability control (DSC);

19. Structured analysis and design technique (SADT).

The main effect of using MES systems are in two areas:

- increase in efficiency by production volumes growth, by increasing the effective loading of equipment, and reducing the production cycle time;

- quality improvement by reducing the amount of defects, inappropriate or requiring re-processing of products, ability to control production and compliance with technological regulations at all stages of production before shipment to the final consumer.

MES systems allow us to bring a significant share of organization and order into production processes, however, if they do not provide modeling and unification capabilities for user interaction, their potential for use may be limited and not provide all the benefits. Using a model-oriented control system for modern production allows us to fully reveal the potential of increasing production efficiency due to the following (Sazonov, Vasilyeva \& Mikhailova, 2020):

- reduce labor costs for the implementation of MES system and obtain first results;

- digital transformation of task and operations management: creating a "digital double" of production processes to provide further control over production execution, standardization and use of best industrial practices, both within a single production site and within geographically distributed holdings, including many production units;

- unification of user interaction, the ability to customize and deploy the solution in accordance with the needs of production, reuse the best practices adopted by the enterprise within other sites, taking into account the features of their work.

The criterion for deciding about the modernizing of the production system is minimizing the transformation costs: it is clear that when simulated they are minimal (due to increased labor productivity and the release of workers). However, the simulation version of the modernization of the production system does not allow the development of an industrial enterprise as an economic system and does not take into account the level of resources available to the enterprise. 


\section{Method}

The problems of developing a methodology and choosing practical tools for digital transformation of enterprises are increasingly becoming the subject of scientific discussions at various levels. Many researchers note that in modern conditions a large number of factors influence the results of a high-tech enterprise. On the one hand, the modern consumer places high demands on the quality of product innovations and the timing of their delivery. This requires strict adherence to technological and organizational standards of production. On the other hand, the activity of a high-tech enterprise takes place in the external environment with a high level of uncertainty and is characterized by the influence of a significant number of disturbing factors. The management of a high-tech enterprise, making decisions, has the need to process large amounts of data that are not always structured, reliable and consistent. Manually processing such arrays is almost impossible. Therefore, it is necessary to create a single information space of the enterprise, where integrated information systems will function. In such environment, the coordination of the functioning of the links of a high-tech enterprise is achieved by creating hierarchical management structures that combine:

- computer methods and tools for translating information into a human-readable form and data mining Business Intelligence (BI);

- BPM systems that implement the concept of process management, in which business processes are considered as a special resource of the enterprise;

- ERP systems that integrate production, intellectual, financial and material asset management strategies of a high-tech enterprise;

- MES systems that allow effectively solving the tasks of operational calendar planning;

- industrial control systems (APCS), which make it possible to automate process control.

Modern high-tech automation systems (BI, ERP, MES, APCS) and control levels can be effectively correlated with each other (Figure 2). Following the data presented below, of the BI and ERP systems form the strategic management level, MES systems determine the tactical level, and APCS determine the operational management level (Simonova \& Khisamutdinov, 2016). In the structure shown in Figure 2, ERP systems provide management efficiency at a strategic level, implementing the functional strategies of the enterprise as a single system. This primarily concerns logistics, finance and intellectual capital. The use of ERP systems optimizes the resource supply of the enterprise. Analytical data processing there is carried out using interactive analytical processing technologies. These technologies provide the preparation of aggregated information based on large data arrays. OLAP technologies are components of BI class software solutions and are implemented by a high-tech enterprise based on automated BI systems.

Effective management at the production level of a high-tech enterprise is provided by MES systems that implement a wide range of functions, from order formation to the release of product innovations. The use of this class of systems makes it possible to solve the tasks of operational calendar management: planning production processes, their documentation, optimization, control, synchronization of technological operations, coordination of production and sales volumes. MES system, being a component of an integrated information and computing system, makes it possible in real time to effectively manage production resources, increasing the performance of a high-tech enterprise. The integration of MES systems into the digital space of a high-tech enterprise generates a number of competitive advantages. In particular, such properties of the MES system as a quick response to deviations of process parameters from standard values, the presence of mathematical tools to minimize deviations in the work schedule, can minimize the duration of the production cycle and the cost of product innovations. ERP-system forms the strategy for fulfilling the order portfolio of a high-tech enterprise, setting the volumes and deadlines for the release of product innovations, and MES system forms the tactics of managing product innovation, while implementing the monitoring function of the production process. In highly automated production, MES system becomes the core of the integration of information business processes. This primarily relates to interaction with various systems, for example, with systems providing resource supply chain planning (SCM systems), product innovation sales and service management (SSM systems), etc. Interacting with the process control system, MES system, providing monitoring of technological processes, allows identifing the key points of formation of the quality of product innovations. 


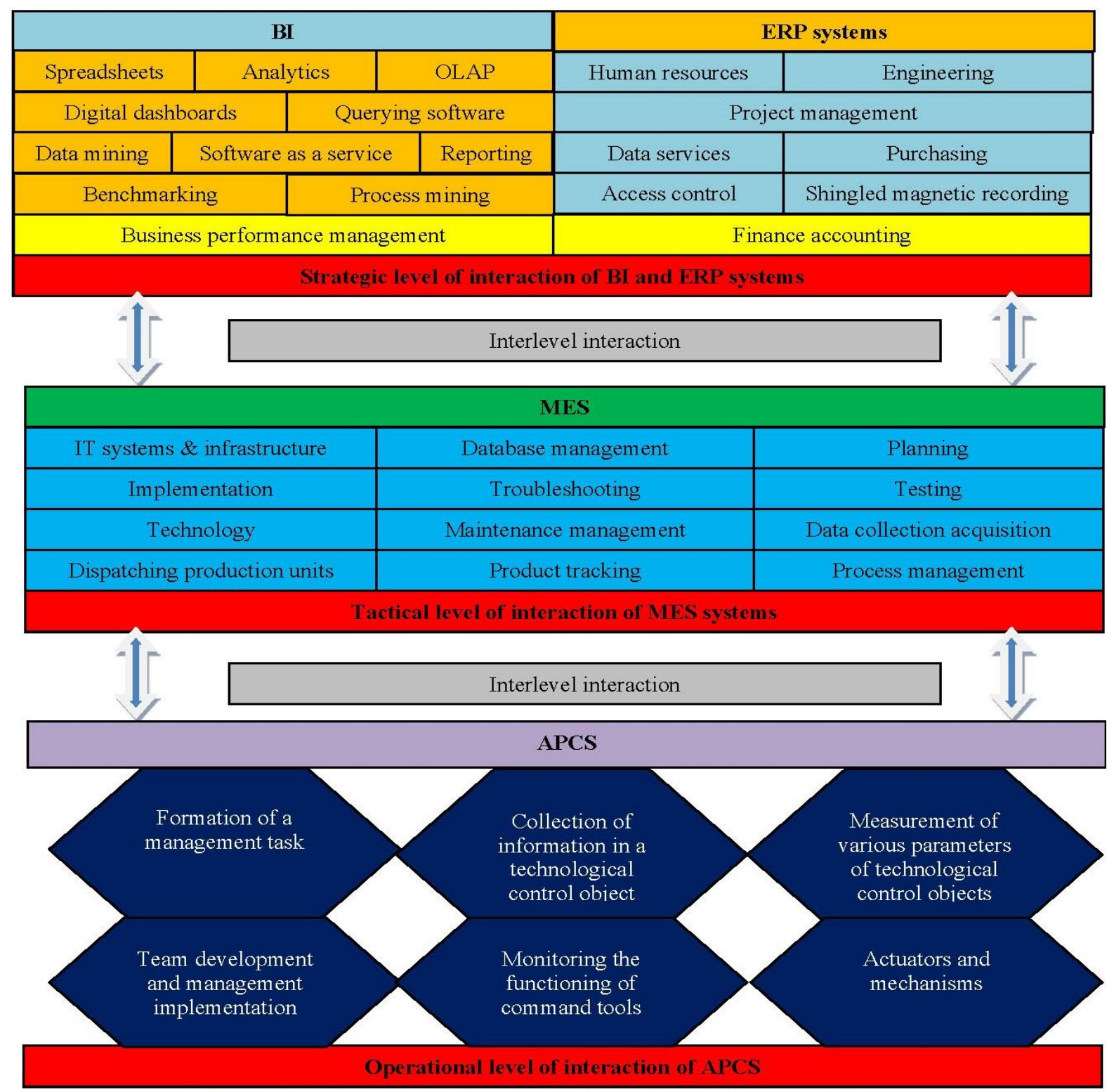

Figure 2. Technological levels of automation of the production process

The level of APCS is a structural complex consisting of certain software and hardware designed to carry out processes related to the automation of control of certain technological equipment at industrial / machine-building enterprises. As a rule, there are control, information, and auxiliary functions of APCS, which allow effective regulation of technological process variables, program control of a group of equipment, technological modes or individual sections of processes, as well as the monitoring and measurement of technological parameters of processes. MES level forms a certain automated system for managing the production activities of a machine-building enterprise, which allows real-time planning, optimization, control, and documentation of production processes from the formation of an order to the release of finished products. MES systems have such functions as: monitoring the status and distribution of resources, operational / detailed planning, production scheduling, managing the quality of products manufactured by an enterprise, managing production processes, equipment maintenance and repair, and analyzing and evaluating performance (Zotova, 2019).

The level of ERP systems allows implementing a strategy related to the integration of logistics (procurement, production, sales), financial (debtors, creditors, banks), and staff functions of enterprises, focused on optimizing the resources of the enterprise through specialized software. The majority of ERP systems process transaction data and 
belong to a special class of online transactional processing (OLTP) systems. Analytical processing of transactional data collected by means of ERP systems is carried out at OLAP level, using automated BI systems. The combined use of the above levels of automation forms a holistic information environment of the enterprise. Table 1 presents the main characteristics of automation systems.

Table 1. Basic automation systems

\begin{tabular}{|c|c|c|c|c|}
\hline $\begin{array}{c}\text { Automation } \\
\text { system }\end{array}$ & $\begin{array}{c}\text { Time horizon } \\
\text { planning }\end{array}$ & $\begin{array}{c}\text { Information } \\
\text { processing frequency }\end{array}$ & Automation processes & Planning features \\
\hline$B I(O L A P)$ & - & Every day & Getting analytical reporting & - \\
\hline$E R P(O L T P)$ & $\begin{array}{l}\text { Week, month, } \\
\text { quarter }\end{array}$ & $\begin{array}{l}\text { Every day / every } \\
\text { week }\end{array}$ & $\begin{array}{l}\text { Administrative and } \\
\text { economic resources }\end{array}$ & $\begin{array}{c}\text { Strategic planning, } \\
\text { taking into account } \\
\text { volumetric } \\
\text { production planning }\end{array}$ \\
\hline MES & $\begin{array}{l}\text { Hour, shift, } \\
\text { week }\end{array}$ & Real time planning & Production processes & $\begin{array}{l}\text { Operational planning } \\
\text { of production process }\end{array}$ \\
\hline$A P C S$ & - & Real time function & $\begin{array}{l}\text { Processing and analysis of } \\
\text { various technological } \\
\text { information }\end{array}$ & - \\
\hline $\begin{array}{l}\text { Flow } \\
\text { management } \\
\text { solution }\end{array}$ & - & Real time planning & $\begin{array}{l}\text { Complete product } \\
\text { manufacturing cycles }\end{array}$ & $\begin{array}{l}\text { Significant increase } \\
\text { in labor productivity }\end{array}$ \\
\hline
\end{tabular}

The level of APCS set by the programmable logic controllers, supervisory control and data acquisition (SCADA) systems, and databases allows the permanent collection and processing of various technological data in real time. The processed information is transmitted to the level of MES systems and can be used to organize the operational management of the production process, taking into account the characteristics of interchangeability and readjustment of equipment. The operational production plan of this level is correlated with the results of the ERP systems for strategic planning and management of administrative and business operations of the enterprise. Consolidated analytical reporting obtained based on the processing of transactional data of the ERP level will determine the last automation step by means of BI systems (Galimova, 2019).

Implementation of MES systems at sites using Kalashnikov Concern as an example.

The development and implementation of MES systems at Kalashnikov Concern sites started in 2015, almost synchronously with the start of ERP, master data management (MDM) and several others. The company at that moment faced challenges associated with a significant increase in production volumes and the need to reduce costs. The analysis of the MES systems market carried out at that time convinced us that the products presented did not allow solving the whole range of production and management tasks. It was mainly on the Russian market that SCADA systems with functional add-ons were presented in the form of an indication of the reasons for downtime by machine operators. Therefore, it was decided to develop their system for specific production conditions, while thinking about the possibility of replication. The system has developed and continues to evolve according to the following scheme: $\{$ Real business task $\} \rightarrow$ Analysis $\} \rightarrow\{$ Development $\} \rightarrow\{$ Testing $\} \rightarrow$ Release $\}$. It should be noted that after three years, the company's management is increasingly convinced that this was an absolutely right decision. There were the following important administrative decisions at the start of the project:

- consolidation of repair services;

- providing the most convenient and acceptable opportunity to any employee of the enterprise to initiate a downtime signal of any equipment;

- translation of all service requests in the digital form;

- introduction of total staff training to work with the system.

A set of only these actions allowed reducing the unplanned downtime of equipment at the enterprise with a fleet of 3 000 units in 1.5 years during the active phase of the project. Practice shows that the introduction of only ERP systems in the enterprise does not allow solving the problem of operational re-planning of production due to the specifics of the systems. As a rule, the introduction of ERP without the MES level is accompanied by an increase in the number of staff involved in manual data entry into the system. MES solutions, on the contrary, are aimed at 
enabling operators to independently enter data into the system, aggregate, and transfer it to external systems (including back to ERP) for analysis and construction of the required reporting. The implementation of MES also solves the important problem of reconciling regulatory and reference information, which is very relevant for the post-Soviet industry in general and engineering in particular. At the Kalashnikov sites, this goal was one of the priorities. Knowing the actual data on the complexity of technological operations and the consumption of cutting tools allows building a process of cyclic verification and optimization of labor costs and consumption of basic materials. MES also allowed building sound management of equipment loading and to establish control over planned and unplanned downtime. From the usual scheme with solid shift transfer logs, production switched to a system of signals and requests for repairs.

During the design, development, and implementation of the system, a solution was created that allows taking all production processes under control: from the creation of production operational plans to monitoring the implementation of tasks by operators and detailed (piece) accounting. The system is built in such a way that each participant in the production process, from the operator to the dispatcher, has an interface corresponding to his role, accessible through electronic terminals (kiosks), industrial tablets in workshops and personal computers. Another conceptual solution is maximum actions in the system with clicks on the touch screen: for example, the operator enters only the number of parts performed per shift, everything else can be selected from the list of tiles on the screen. This allowed us to minimize the risks of rejection of a new IT solution at the implementation and support stage. The system greatly simplifies the work of employees: the operator receives tasks in accordance with the adopted plan, can view drawings and 3D-models of assembly unit details, notify about the need for repair, adjustment, tool replacement, and other downtimes. The head of the site or workshop receives a tool to control the production process and prompt response to changes.

The system implements various operating modes that ensure a gradual increase in the culture of production processes from "chaos" to shift-daily tasks and automatic planning. Thanks to this approach, MES can be used in enterprises with different levels of production culture. The system does not have high input requirements for existing business processes in the company. The company can gradually improve them with the introduction of the system. For example, in individual production areas, the automatic planning mode may be introduced, while in other areas, the manual distribution of shift-daily tasks continues to operate without internal conflicts. The system takes up most of the tasks for calculating production schedules. The scheduler application is designed to solve the problem of optimal planning of the work flow on machines. It is built in the form of a separate server, perceiving at the input a task for planning, changing a task, launching it for calculation, and obtaining results. A server can handle many tasks at the same time. This approach allows us to reduce equipment downtime and the time spent on repair and commissioning, and these are precisely the key factors that determine the efficiency of the production process and the rate of return on expensive equipment.

In the calculation process, the scheduling task is solved for the deterministic case of many machines working to complete the entire number of things. For details and tasks, a launch and / or launch / release plan may be indicated, equipment availability may be limited at a given time. In addition, we can specify a calendar plan for shifts (times of availability for work). In the general case, a task of this type has exponential complexity with respect to the number of tasks and machines. By using certain restrictions and heuristic policies to apply the rules, we managed to build and implement a polynomial solution. All optimizations are taken into account in the intermediate sorting of job candidates for placement on machines, which is carried out at each step of the algorithm. This allowed us to build an algorithm that works on a real task (task planning for one site for a month) for no more than 1-2 seconds. Based on the created algorithm, more complex methods of working with the schedule are implemented, in particular, genetic development or a dynamic re-planning mode, if necessary (failure of the machine, etc.). This means that at each step an analysis and schedule change for actual events is performed. The main directions of the system's long-term development are the following: end-to-end planning module (1Q2019), machine learning, and interactive assistance in the formation of the operational plan (2Q2019). The confirmed effects from the introduction of MES and related systems (Service Desk, BI) include an increase in the average load of computer numerical control machines by $12-15 \%$ and refusal to unreasonably acquire two new milling machines in 1.5 years of the active phase of the project.

\section{Conclusion}

A study of the digital transformation tools of a high-tech enterprise by informing business processes and creating robotic organizational and production structures, which are the basis for the effective implementation of the enterprise development strategy, showed that it is advisable to create information systems as hierarchical structures for managing automated production, covering strategic (BPM and ERP systems) and tactical (MES systems) management levels, as well as the operational calendar level management (APCS). In order to bring the automation level of the control and production subsystems into conformity with the created organizational and production links, it is necessary to integrate industrial robotics. 
The state strategy of the Russian Federation is aimed at implementing digital transformation mechanisms, therefore, high-tech enterprises are fully aware that they cannot be avoided, and they will, to a large extent, be conditions for the growth of their competitiveness in the domestic and foreign markets.

Russian high-tech enterprises do not see global opportunities for digital transformation and are not ready for full integration into external digital space. The main reason is the unpreparedness of enterprises to implement open innovations, free distribution of the results of their intellectual activities, as well as the sharing of assets.

The transformation of the existing business model is one of the most important components of the digital maturity growth of a high-tech enterprise and, as a rule, is carried out in the direction of the consistent expansion of various cooperative interactions, use of multilateral platforms, and creation of business ecosystem.

The use of the integration module of information systems operating on different platforms involves the transition from creating a single integrated information space to a more advanced and efficient technological solution, which, of course, includes eliminating the functioning of information systems, which require a large amount of computing resources and staff.

For the successful implementation of the procedure for integrating data obtained from the operation of information systems, it is necessary to create a bi-directional high-tech integration scheme at machine-building enterprise.

There are basic stages of integration development. It is also proposed to use a modified structure, characteristic feature of which is the presence in it of the so-called "intelligent agents", which are specialized software modules that are controlled by a special data exchange protocol. Modules also have the ability to implement synchronization mechanisms with business objects and provide the technical ability to integrate three or more different information systems.

\section{References}

Ananyin, V. I., Zimin, K. V., Lugachev, M. I., Gimranov, R. D., \& Skripkin, K. G. (2018). Digital Enterprise: Transforming into a New Reality. Business Informatics, 2(44), 45-54.

Dmitriev, O. N., \& Novikov, S. V. (2019). Economic Optimization of the Modular Structure of Complex Objects. Russian Engineering Research, 39(6), 503-506.

Galimova, M. P. (2019). Readiness of Russian enterprises for digital transformation: organizational drivers and barriers. Bulletin of the Ufa State Oil Technical University. Science, education, economics, 1(27), 27-37.

Gileva, T. A. (2019). Digital Maturity of an Enterprise: Assessment and Management Methods. Bulletin of the Ufa State Oil Technical University. Science, education, economics, 1(27), 38-52.

Ilyin, I. V., Levina, A. I., \& Dubhorn, A. S. (2019). Digital transformation as a factor in the formation of architecture and enterprise IT architecture. Economics and Environmental Management, 3, 50-55.

Koch, T., \& Windsperger, J. (2017). Seeing through the network: Competitive advantage in the digital economy. Journal of Organization Design, 6, 6.

Orekhova, S. V. (2018). Industrial enterprises: electronic vs. traditional business model. Space of Economics, 4, 77-94.

Savich, Yu. A. (2018). Digital transformation and its impact on the competitiveness of industrial enterprises. ECONOMINFO, 4, 44-48.

Sazonov, A. A., Vasilyeva, I. A., \& Mikhailova, L. V. (2020). The study of management mechanisms of domestic industrial enterprises in the context of a new technological concept. Bulletin of Moscow State Regional University. Series: Economics, 1, 74-81.

Simonova, L. A., \& Khisamutdinov, M. R. (2016). Import substitution in the task of integrating PLM, ERP, and MES information systems. Basic Research, 12(2), 338-343.

Zotova, E. A. (2019). Integration of ERP and MES systems. Young Scientist, 4, 1-3.

\section{Copyrights}

Copyright for this article is retained by the author(s), with first publication rights granted to the journal.

This is an open-access article distributed under the terms and conditions of the Creative Commons Attribution license (http://creativecommons.org/licenses/by/4.0/). 